\title{
Tuberculosis abdominal: Patología infrecuente en un paciente joven. Reporte de un caso
}

\author{
Caterin Arévalo ${ }^{1}$, Jhon Rosales ${ }^{1}$, Diego Lozano ${ }^{1}$, \\ Nayib Zurita ${ }^{1}$ y Bernardo A. Borráez-Segura ${ }^{2}$
}

\begin{abstract}
Abdominal tuberculosis: An unusual disease in a young patient. A case report
Introduction: Abdominal tuberculosis is a reemerging problem and is one of the most important communicable diseases in the world. Despite expectations about the eradication in developing countries, it has recently been re-declared as a global emergency pathology. The increased incidence and prevalence shows an abdominal shape as one of the most common extrapulmonary involvement presentations. Objective: Since tuberculosis can affect various organs, it has a wide range and spectrum of signs and symptoms that make diagnosis difficult and delay treatment. Therefore, this review of the topic is done, concentrating on the fact that the high suspicion index should be an important factor in the early diagnosis. Treatment can be initiated helping to prevent and reduce high morbidity and mortality rates. Case Report: We present a case of a young patient with ascites secondary to abdominal tuberculosis confirmed by biopsy and increased adenosine deaminase in the peritoneal fluid. The main clinical findings, paraclinic, imaging studies and treatment are described.
\end{abstract}

Key words: tuberculosis; ascites; adenosine deaminase.

\section{Resumen}

Introducción: La tuberculosis abdominal es un problema reemergente, y es una de las enfermedades transmisibles más importante en todo el mundo. A pesar de las expectativas acerca de su erradicación en países en desarrollo, ha sido recientemente declarada de nuevo como una patología de emergencia mundial. Con el aumento de su incidencia y prevalencia, su forma abdominal es una de las presentaciones de afectación extrapulmonar más comunes. Objetivo: Dado que la tuberculosis puede afectar diversos órganos, tiene una amplia gama y gran espectro de signos y síntomas que dificultan su diagnóstico y retrasan el tratamiento. Por esto, se realiza esta revisión de tema, concentrándonos en que el alto índice de sospecha debe ser un factor importante en el diagnóstico precoz, para que una vez establecido, se pueda iniciar el tratamiento ayudando a prevenir y disminuir las altas tasas de morbilidad y mortalidad evidenciadas en la actualidad. Caso Clínico: Paciente joven con presencia de ascitis secundaria a tuberculosis abdominal confirmada por una biopsia y el aumento de la adenosin deaminasa en el líquido peritoneal. Se describen los principales hallazgos clínicos, paraclínicos, estudios imagenológicos y tratamiento.

Palabras clave: tuberculosis; ascitis; adenosin deaminasa.
Universidad del Sinú. Cartagena de Indias. Colombia.

¿Departamento de Cirugía Gastrointestinal, Instituto Colombiano de Cancerología, Bogotá, Colombia.

Recibido el 30 de marzo y aceptado para publicación el 7 de junio de 2018.

Correspondencia a: Dra. Caterin Arévalo Zamora cate-90@hotmail.com

\section{Introducción}

La tuberculosis peritoneal (TBP) es una enfermedad difícil de diagnosticar que puede simular patologías malignas. Se presentan en un notable porcentaje de pacientes $(10 \%)$ que ingresan al Servicio de Urgencias con abdomen agudo siendo el peritoneo una de las localizaciones extrapulmonares más comunes $^{1,2}$. Con el incremento de la prevalencia del virus de la inmunodeficiencia humana (VIH) en los países desarrollados, la tuberculosis (TB) debe ser considerada como parte del diagnóstico diferencial en los pacientes con ascitis y/o masas abdominales o pélvicas ${ }^{2}$.

A continuación, reportamos un caso de TBP en un paciente joven con ascitis. Se describen los principales hallazgos clínicos, paraclínicos e intraoperatorios y se presenta una revisión de la literatura. 


\section{Presentación del caso}

Paciente hombre de 24 años, con cuadro clínico de 6 meses de evolución consistente en dolor abdominal generalizado de predominio en región mesogástrica e hipogastrio, intermitente, asociado a picos febriles no cuantificados y pérdida de peso progresiva. Desde hace 10 días, refiere dolor abdominal persistente agudizado, sin mejoría con la toma de medicamentos, con las mismas características anteriores. Los antecedentes personales son el consumo de sustancias estupefacientes (marihuana, cocaína).

Al examen físico, se encuentra un paciente en mal estado nutricional, con taquicardia y disnea

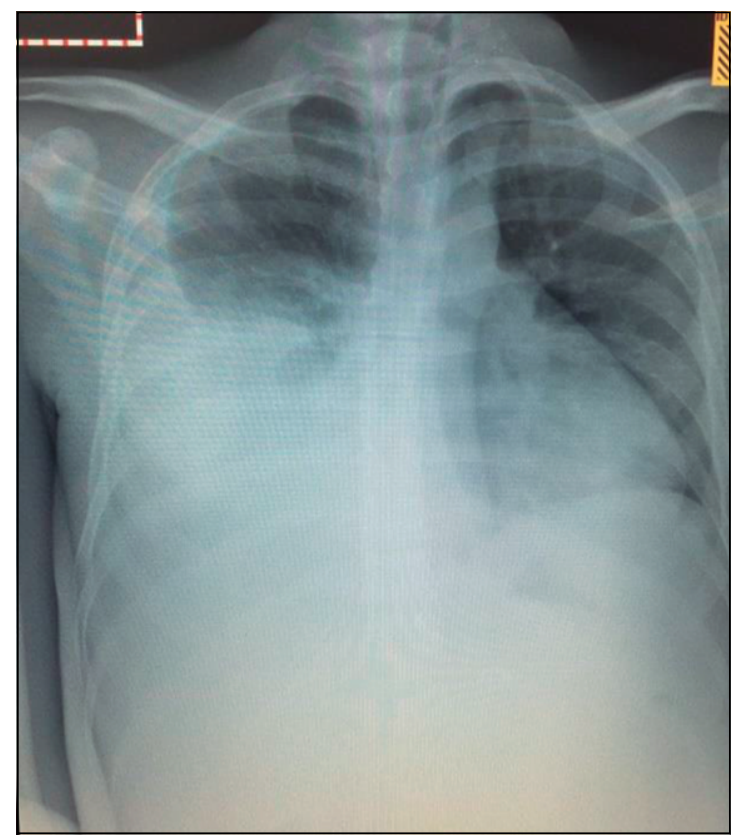

leve. A la exploración física, se encuentra el abdomen distendido con dolor en hipogastrio y fosa iliaca izquierda, sin signos de irritación peritoneal con onda ascítica positiva. El paciente, es admitido en el Servicio de Urgencias donde se realizan estudios de laboratorio iniciales que evidencian leucocitosis, neutrofilia, alteración hidroelectrolítica dada por hiponatremia y falla renal aguda. Serología para VIH negativa y baciloscopia seriada de esputo negativa. Dentro de los estudios complementarios practicados, se realizó:

- Esófago-gastroduodenoscopia: Gastritis crónica moderada.

- Colonoscopia total: Dentro de límites normales.

- Radiografía de tórax: Opacidad homogénea que ocupa la mayor parte del hemitórax derecho dado principalmente, por derrame pleural que borra el contorno derecho y el hemidiafragma del mismo lado (Figura 1).

- Ecografía abdominal con agenesia renal derecha, moderada ectasia izquierda y derrame pleural derecho con atelectasias basales posteriores.

- Tomografía abdominal contrastada, con presencia de derrame pleural derecho y ascitis (A), riñón único izquierdo con ectasia piélica (B). Proceso peritoneal difuso de origen inflamatorio o neoplásico $(\mathrm{C})$, no descarta plastrón inflamatorio en la mitad derecha del hueco pélvico (Figura 2).

Dados hallazgos clínicos e imagenológicos de ascitis, se iba a programar la realización de una paracentesis diagnóstica para establecer la etiología de la enfermedad, sin embargo, durante este tiempo, el paciente presenta un deterioro en el estado funcional, disnea y signos de irritación peritoneal, por lo cual, es llevado a la realización de una laparoscopia diagnóstica. Dado el acceso limitado a la cavidad

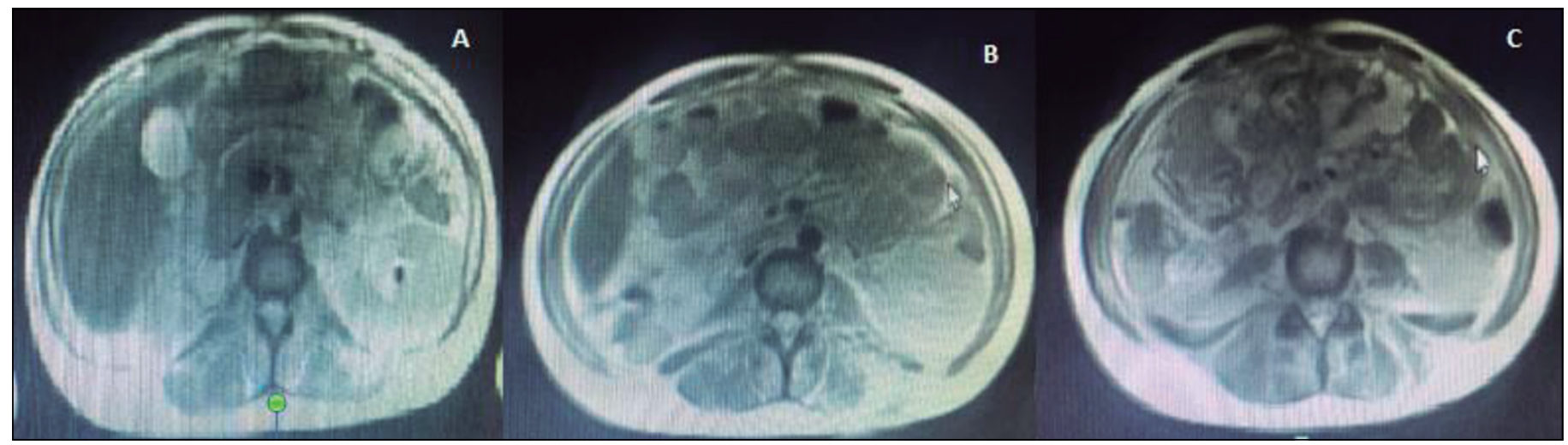

Figura 2. Tomografía Abdominal Contrastada, con presencia de derrame pleural derecho y ascitis (A), riñón único izquierdo con ectasia piélica (B). Proceso peritoneal difuso de origen inflamatorio o neoplásico (C), no descarta plastrón inflamatorio en la mitad derecha del hueco pélvico. 
abdominal, se decide convertir a laparotomía, donde se encuentran múltiples adherencias peritoneales (A), se confirman hallazgos imagenológicos con moderado líquido ascítico, se toma muestra para cultivo y se evidencian lesiones granulomatosas en epiplón y peritoneo (B) de las cuales se toman biopsias (Figura 3).

El procedimiento finaliza sin complicaciones, y el paciente no requiere hospitalización en Unidad de Cuidados Intensivos, es trasladado a salas de recuperación, con posterior evolución satisfactoria, se inicia dieta a tolerancia a los 5 días del posoperatorio y se avanza a dieta normal hasta su egreso. Se da de alta con recomendaciones generales y signos de alarma, cita control con cirugía general y manejo analgésico ambulatorio a necesidad, sin antibioticoterapia.

En controles, se reciben resultados de la biopsia peritoneal, la cual reporta granulomas caseificantes y aumento de los niveles de adenosin deaminasa del líquido ascítico con un valor de 201,2 UI/L. Lo que permitió realizar el diagnóstico, de tuberculosis abdominal, iniciando así el tratamiento antituberculoso según las guías gubernamentales e institucionales (isoniazida, rifampicina, pirazinamida y etambutol) con adecuada evolución y mejoría de su sintomatología. Se decide citar a controles cada 3 meses; el paciente asiste a los mismos, finaliza el tratamiento posteriormente, con evidencia clínica de la remisión total de todos los síntomas.

\section{Discusión}

\section{Epidemiología}

La primera referencia de posible TBP fue hecha en 1643 cuando la autopsia de Louis XIII mostró unas lesiones intestinales ulcerativas asociadas a una gran cavidad pulmonar ${ }^{3}$. Con el paso de los años y el descubrimiento de medicamentos efectivos contra el Mycobacterium tuberculosis, en los países desarrollados cambió el patrón de presentación con una mayor prevalencia de formas extrapulmonares que incluían la TB abdominal ${ }^{4}$.

En Colombia, la incidencia estimada para el 2011 fue de 24,5 por 100.000 habitantes y si bien en la última década se ha observado una disminución en el número de casos nuevos, el porcentaje de la enfermedad extrapulmonar ha aumentado de 14 a $20 \%{ }^{5}$.

La TB es un problema de salud pública en regiones endémicas y debido al fenómeno de la migración y al incremento del uso de la terapia con inmunosupresores la enfermedad resurge en las regiones donde ya había estado controlada ${ }^{6}$.

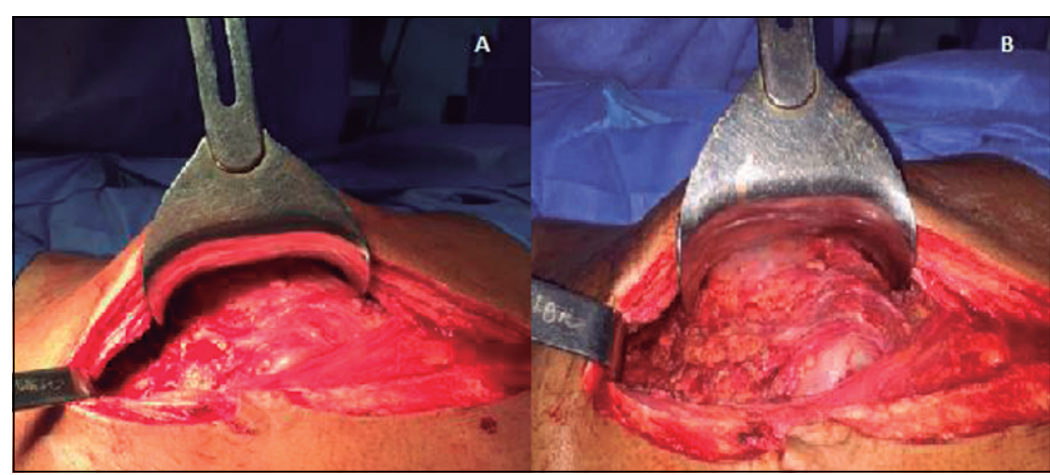

Figura 3. Hallazgos intraoperatorios de laparotomía exploratoria donde se encuentran múltiples adherencias peritoneales (A), moderado líquido ascítico con lesiones granulomatosas en epiplón y peritoneo.

\section{Patogénesis e histopatología}

El microorganismo alcanza el tracto gastrointestinal mediante cuatro vías de propagación: En la primera, la capa mucosa del tracto gastrointestinal puede estar infectada con los bacilos con la formación de tubérculos epitelioides en el tejido linfoide de la submucosa. Después de 2 a 4 semanas, la necrosis caseosa de los tubérculos conduce a la ulceración de la mucosa que más tarde se puede propagar en las capas más profundas, en los ganglios linfáticos adyacentes y en el peritoneo ${ }^{7}$. La segunda vía es la diseminación hematógena del foco tuberculoso de otra parte del cuerpo: los riñones, ganglios linfáticos y el peritoneo. La tercera vía de propagación directa incluye al peritoneo a partir de focos adyacentes infectados, incluyendo las trompas de Falopio o sus anexos, absceso del psoas, o las secundarias a espondilitis tuberculosa. Por último, se puede propagar a través de los canales linfáticos infectados.

La patología se caracteriza por úlceras, fibrosis, engrosamientos y estenosis de la pared intestinal, aumento de tamaño de ganglios linfáticos mesentéricos, engrosamiento omental y tubérculos peritoneales siendo su localización más frecuente la región ileocecal ${ }^{8}$.

\section{Presentación clínica}

Predominantemente, es una enfermedad de adultos jóvenes; dos terceras partes de los pacientes tienen entre 21 y 40 años, y la incidencia por género es igual o con algún predominio del género masculino, aunque algunos informes mencionan que afecta a ambos sexos por igual ${ }^{9}$.

El cuadro clínico corresponde a su localización, y según la literatura, Banshani et $\mathrm{al}^{10}$, observó una malabsorción franca en el $21 \%$ de los pacientes, mientras que Tandon et $\mathrm{al}^{11}$ informó obstrucción 
intestinal en el 75\% de los pacientes. También se ha notificado hemorragia rectal en algunos casos.

Las manifestaciones sistémicas de la infección tuberculosa incluyen fiebre baja de predominio nocturno, malestar, diaforesis, anorexia y pérdida de peso, éstas más frecuentes en lesiones intestinales ulcerosas y tuberculosis peritoneal con ascitis.

Se caracteriza por diferentes modos de presentación: Crónica, aguda y aguda sobre la crónica, o como un hallazgo incidental. Además, ocurre en tres formas:

- Húmeda con ascitis.

- Seca con adherencias.

- Fibrótica con engrosamiento de epiplón y ascitis loculados $^{12}$.

Asimismo, puede aparecer como: linfadenopatía tuberculosa, TBP, gastrointestinal y la visceral que afecta a los órganos sólidos ${ }^{13}$. En general, los jóvenes con TB abdominal se presentan clínicamente con un cuadro de fiebre, dolor abdominal, anorexia, pérdida de peso y ascitis en más del $80 \%$ de los $\operatorname{casos}^{14}$. Sin embargo, ninguno de estos síntomas es específico para la enfermedad, dificultando su diagnóstico y haciendo de ésta un reto por su forma de presentación, su variabilidad y la limitación en la disponibilidad de test diagnósticos.

\section{Diagnóstico}

Actualmente hay varias pruebas que se usan para confirmar el diagnóstico de la enfermedad:

- Paraclínicos: Las pruebas hematológicas revelan anemia, leucocitosis con linfocitosis relativa. La hipoalbuminemia es frecuente.

- Pruebas serológicas: Actualmente, los más reconocidos son:

- Anticuerpo fluorescente de antígeno y ensayo inmunoabsorbente ligado a enzima: Probable diagnóstico.

- Anticuerpos anti-factor cordón: Diferenciación de la enfermedad de Crohn ${ }^{15}$.

- La prueba de tuberculina (Mantoux o Heaf) fue positiva en la mayoría de los pacientes con diagnóstico, pero tiene un valor limitado porque no diferencia entre la enfermedad activa y la sensibilización previa por contacto o vacunación ${ }^{16}$.

También, hay varios test con diferente sensibilidad y especificidad:

- Laparoscopia diagnóstica: Técnica quirúrgica segura y fácilmente establece el diagnóstico de la TBP, permitiendo tomar una biopsia peritoneal y cultivo del líquido ascítico ${ }^{17}$.

- Biopsia peritoneal: Idealmente por laparoscopia. Método de investigación de elección. Conside- rarlo en pacientes con ascitis inexplicable. La necrosis de caseificación de granulomas en el estudio histológico de la tuberculosis permite hacer el diagnóstico ${ }^{7}$.

- Cultivo del líquido ascítico: Aislamiento del microorganismo, suele ser positivo en un $30-40 \%$ de los casos.

- Citológico del líquido ascítico: El estudio del líquido peritoneal es de tipo exudativo en el $80 \%$ de los casos. La TB se sospecha en líquidos con linfocitos aumentados sin presencia de células malignas y actividad elevada de interferón gamma y adenosin deaminasa con valores mayores a $36 \mathrm{U} / \mathrm{I}^{14}$, además, proteína de más de $3 \mathrm{~g} / \mathrm{dl}$, el cociente entre la glucosa en sangre es inferior a 0,96 y el gradiente sérico de albúmina ascítica es inferior a $1,1 \mathrm{~g} / \mathrm{dl}^{18}$.

- La colonoscopia con biopsia o biopsia hepáti$\mathrm{ca}^{19,20}$ ha sido útil sólo en algunos pacientes.

El diagnóstico definitivo requiere la identificación del bacilo en el líquido ascítico o en el tejido peritoneal $^{12}$.

\section{Diagnóstico diferencial}

Debido a que el lugar más común de afectación en el tracto gastrointestinal es el área ileocecal, su diagnóstico es difícil y se debe distinguir de la enfermedad de Crohn, apendicitis y cáncer. Además, si hay peritonitis, se puede confundir fácilmente con patologías ginecológicas ${ }^{8}$.

Govind K. Makharia et al plantean y validan un score teniendo en cuenta la localización del compromiso del colon (sigmoides), la presencia de sangre en heces, la pérdida de peso y hallazgos histológicos específicos (colitis localizada) como mayores predictores para hacer el diferencial entre enfermedad de Crohn y tuberculosis, logrando una sensibilidad de hasta el $90 \%$ con una especificidad del 60 al $80 \%{ }^{21}$.

\section{Estudios complementarios}

En la actualidad, los avances en las técnicas moleculares han permitido realizar un diagnóstico más exacto y temprano de la enfermedad.

- Interferón gamma: Evalúa la respuesta inmune mediada por células contra el Mycobacterium tuberculosis, mostrando, junto con la adenosina deaminasa, una mayor sensibilidad y especificidad para el diagnóstico positivo de tuberculosis.

- Reacción en cadena de la polimerasa: Es una prueba rápida de amplificación de genes para aislar el microorganismo entre 24 a 48 h. Su uso en la práctica clínica está limitada por el alto costo y baja sensibilidad ${ }^{22}$. 
- Sistema radiométrico BACTEC $^{6}$ : Es un método automatizado que permite la detección más rápida del Mycobacterium en un promedio de 14 a 27 días. Este sistema, reduce el tiempo de aislamiento del cultivo, sin embargo, es más caro que el medio de cultivo sólido y utiliza material radioactivo, lo que genera un problema de manipulación ${ }^{23}$.

- Marcador tumoral CA 125: Niveles séricos altos pueden ser encontrados tanto a nivel sérico en la mayoría de los pacientes con ascitis exudativa de cualquier etiología, pudiéndose confundir con el carcinoma de ovario en etapas avanzadas. Además, su determinación parece útil para evaluar la respuesta terapéutica a los fármacos antituberculo$\operatorname{sos}^{14}$.

\section{Imágenes diagnósticas}

- Radiografía de tórax: Lesiones pulmonares concomitantes se observan en menos del $25 \%$ de los casos.

- Ecografía abdominal: Los resultados no permiten confirmar el diagnóstico debido a la poca especificidad de los hallazgos que por separado no tienen ningún valor ${ }^{14}$. La ascitis es el signo más frecuentemente encontrado, la cual es libre y puede tener ecogenicidad en relación con su naturaleza exudativa ${ }^{24}$. Otros signos sugestivos pueden ser: el engrosamiento del peritoneo, la aglutinación de las asas intestinales entre sí o con la pared abdominal anterior, nódulos peritoneales, adherencias visualizados como estructuras lineales hipoecoicas en tiras finas y flotando y los ganglios linfáticos profundos a menudo descrito como múltiples masas hipoecoicas y a veces confluentes ${ }^{25}$.

- Tomografía abdominal contrastada: Modalidad de elección en la detección de tuberculosis abdominal $^{13}$. Los principales signos sugestivos son: la ascitis (densidad usual de 25 a $45 \mathrm{HU}$ ), ganglios linfáticos con centro hipodenso, engrosamiento del mesenterio, epiplón y peritoneo (regular y uniforme), y la aglutinación de las asas intestinales ${ }^{26,27}$.

\section{Tratamiento}

El tratamiento se hace con medicamentos antituberculosos durante 6 meses en la mayoría de los casos $^{6}$. El protocolo recomendado en la actualidad combina cuatro fármacos: isoniacida, rifampicina, pirazinamida y etambutol, dados 1 vez al día durante 2 meses, continuando 4 meses con la terapia de combinación de isoniacida y rifampicina ${ }^{28}$.

Durante este proceso, el paciente debe estar cuidadosamente monitorizado con pruebas de función hepática, ya que los medicamentos pueden producir disfunción en el hígado, lo que es relativamente infrecuente ${ }^{29}$. Arajhi y cols, realizaron estudios, evidenciando que la administración combinada de corticosteroides y el tratamiento antituberculosis reducían la frecuencia en la morbilidad y complicaciones de los pacientes con tuberculosis abdominal ${ }^{30}$.

En una minoría de pacientes con TB abdominal, la presentación de obstrucción intestinal o perforación es una clara indicación para una cirugía de emergencia; en estos casos, el intestino perforado o estenosado, debe ser resecado y el material debe ser remitido para su estudio histopatológico ${ }^{31}$

\section{Conclusiones}

La TBP es considerada como una enfermedad de inicio insidioso y presentación crónica, y aunque rara vez es una emergencia, sus complicaciones con cuadros agudos, sin un adecuado enfoque clínico, pueden desorientar al médico.

Es por eso que supone un reto diagnóstico importante, dada la variabilidad de presentación y su amplia constelación de síntomas, necesitamos una amplia sospecha clínica y una correlación con los hallazgos radiológicos, asociado al uso de procedimientos mínimamente invasivos (biopsias por radiología intervencionista y paracentesis); incluso, en la actualidad, los avances en las técnicas moleculares, (reacción en cadena de polimerasa, sistema radiométrico, uso de interferón gamma) proporcionan un nuevo enfoque que permitiría abordar la enfermedad en estadios iniciales brindando un mejor tratamiento para la misma, con mejores factores pronósticos para el paciente y evitando en algunos casos el uso de la cirugía de urgencia.

\section{Responsabilidades éticas}

Protección de personas y animales. Los autores declaran que para esta investigación no se han realizado experimentos en seres humanos ni en animales.

Confidencialidad de los datos. Los autores declaran que han seguido los protocolos de su centro de trabajo sobre la publicación de datos de pacientes.

Derecho a la privacidad y consentimiento informado. Los autores han obtenido el consentimiento informado de los pacientes y/o sujetos referidos en el artículo. Este documento obra en poder del autor de correspondencia.

\section{Conflicto de intereses}

Los autores declaran no tener conflictos de interés. 


\section{CASOS CLÍNICOS}

\section{Bibliografía}

1. Mukhopadhyay A, Dey R, Bhattacharya U. Tuberculosis with an acute abdomen: Our clinical experience. J Clin Diagn Res. 2014 Jul;8(7):NC07-9. doi: 10.7860/ JCDR/2014/8654.4574. Epub 2014 Jul 20.

2. Lal N, Soto-Wright V. Peritoneal tuberculosis: Diagnostic options. Infect Dis Obstet Gynecol. 1999;7:244-7.

3. Paustian FF, Bockus HL. So-called primary ulcerohypertrophic ileocecal tuberculosis. Am J Med [Internet]. 1959;27:509-18. Available from: http:// www.sciencedirect.com/science/article/ pii/0002934359900166

4. Mukhtar AU. Ileo-caecal tuberculosis mimicking colonic tumour-case report. Cent Afr J Med [Internet]. 2000;46:44-5. Available from: http://www.ncbi.nlm.nih. gov/pubmed/14674209

5. Solier J, Bolivar A, Calvo LS, Roberto SL. Tuberculosis peritoneal simulando cáncer de ovario Peritoneal tuberculosis simulating ovarian cancer. Acta Médica Colomb. 2014;2008:383-7.

6. Guirat A, Koubaa M, Mzali R, Abid B, Ellouz S, Affes N, et al. Peritoneal tuberculosis. Clin Res Hepatol Gastroenterol. 2011;35:60-9.

7. Akhan O, Pringot J. Imaging of abdominal tuberculosis. European Radiology 2002. p. 312-23.

8. Sharma MP, Bhatia V. Abdominal tuberculosis. Indian J Med Res. 2004; 120:305-15.

9. Chong VH, Lim KS. Gastrointestinal tuberculosis. Singapore Medical Journal 2009;50:638-46.

10. Bhansali SK. Abdominal Tuberculosis: Experiences with 300 Cases. Am J Gastroenterol. 1977;67:324-37.

11. Bhargava DK, Tandon HD, Chawla TC, Tandon BN, Kapur BML. Diagnosis of ileocecal and colonic tuberculosis by colonoscopy. Gastrointest Endosc. [Internet]. 1985;31:68-70. Available from: http://www.sciencedirect.com/science/ article/pii/S0016510785719955

12. Mimidis K, Ritis K, Kartalis G. Peritoneal tuberculosis. Annals of Gastroenterology 2005; 18:325-9.

13. Debi U, Ravisankar V, Prasad KK, Sinha SK, Sharma AK. Abdominal tuberculosis of the gastrointestinal tract: Revisited. World Journal of Gastroenterology 2014;20:14831-40.

14. Verspyck E, Struder C, Wendum D, Bourgeois D, Lariven S, Marpeau L. [Peritoneal tuberculosis]. Ann Chir [Internet]. 1997;51:375-8. Available from: http://www.ncbi.nlm.nih.gov/ pubmed/9297863

15. Kashima K, Oka S, Tabata A, Yasuda K, Kitano A, Kobayashi K, et al. Detection of anti-cord factor antibodies in intestinal tuberculosis for its differential diagnosis from Crohn's disease and ulcerative colitis. Dig Dis Sci. 1995;40:2630-4.

16. Palmer KR, Patil DH, Basran GS, Riordan JF, Silk DB. Abdominal tuberculosis in urban Britain-a common disease. Gut 1985;26:1296-305.

17. Al-Mulhim AA. Laparoscopic diagnosis of peritoneal tuberculosis. Surgical Endoscopy and Other Interventional Techniques. 2004. p. 1757-61.

18. Wilkins EGL. Tuberculous peritonitis: Diagnostic value of the ascitic/blood glucose ratio. Tubercle 1984;65:47-52

19. Breiter JR, Hajjar JJ. Segmental tuberculosis of the colon diagnosed by colonoscopy. Am J Gastroenterol [Internet]. 1981;76:369-73. Available from: http://www.embase.com/search/re sults?subaction=viewrecord \& from $=$ expo rt\&id=L12203053\%5Cnhttp://sfxhosted. exlibrisgroup.com/dal?sid=EMBASE\&iss $\mathrm{n}=00029270 \& \mathrm{id}=$ doi:\&atitle=Segmental + tuberculosis + of + the + colon+diagnosed + by + colonoscopy\&stitle $=$ AM. + J.+GASTRO ENTEROL.\&ti

20. Desai CS, Josh AG, Abraham P, Desai DC, Deshpande RB, Bhaduri A, et al. Hepatic tuberculosis in absence of disseminated abdominal tuberculosis. Ann Hepatol. 2006;5:41-3.

21. Yu H, Liu Y, Wang Y, Peng L, Li A, Zhang Y. Clinical, endoscopic and histological differentiations between Crohn's disease and intestinal tuberculosis. Digestion 2012;85:202-9.

22. Diagnostics N, Group W, Partnership STB. Pathways to better diagnostics for tuberculosis. Extranetwhoint [Internet]. 2009;1-151. Available from: http://apps. who.int/tdr/publications/tdr-researchpublications/tb-blueprint/pdf/pathways- tuberculosis.pdf

23. Sanai FM, Bzeizi KI. Systematic review: tuberculous peritonitis-presenting features, diagnostic strategies and treatment. Aliment Pharmacol Ther [Internet]. 2005;22:685-700. Available from: http:// www.ncbi.nlm.nih.gov/pubmed/16197489

24. Rodríguez E, Pombo F. Peritoneal tuberculosis versus peritoneal carcinomatosis: distinction based on CT findings. J Comput Assist Tomogr [Internet]. 2006;20:269-72. Available from: http://www.ncbi.nlm.nih.gov/ pubmed/8606235

25. Ramaiya LI, Walter DF. Sonographic features of tuberculous peritonitis. Abdom Imaging [Internet]. 1993;18:236. Available from: http://www.ncbi. nlm.nih.gov/entrez/query.fcgi? $\mathrm{cmd}=\mathrm{Re}$ trieve $\& \mathrm{db}=$ PubMed $\&$ dopt $=$ Citation $\&$ li st uids $=8431688$

26. Cheung HYS, Siu WT, Yau KK, Yang GPC, Li MKW. Abdominal tuberculosis mimicking metastasis in a patient with carcinoma of the oesophagus. Asian journal of surgery Asian Surgical Association. 2006;29:49-50.

27. Lee DH, Lim JH, Ko YT, Yoon Y. Sonographic findings in tuberculous peritonitis of wet-ascitic type. Clin Radiol. 1991;44:306-10

28. Saigal S, Nandeesh HP, Sarin SK CN - FROM CIDG REGISTER MEDLINE 21479583 ASR. Safety of an ofloxacin-based antitubercular regimen for the treatment of tuberculosis in patients with underlying chronic liver disease: a preliminary report. J Gastroenterol Hepatol. 2001;16:1028-32.

29. Sefr R, Rotterova P, Konecny J. Perforation peritonitis in primary intestinal tuberculosis. Dig Surg. 2001;18:475-9.

30. Alrajhi AA, Halim MA, Al-Hokail A, Alrabiah F, Al-Omran K. Corticosteroid treatment of peritoneal tuberculosis. Clin Infect Dis. 1998;27:52-6.

31. Chou CH, Ho MW, Ho CM, Lin PC, Weng CY, Chen TC, et al. Abdominal tuberculosis in adult: 10-year experience in a teaching hospital in central Taiwan. J Microbiol Immunol Infect [Internet]. 2010;43:395-400. Available from: http://dx.doi.org/10.1016/S16841182(10)60062-X 